Sharif University of Technology
Scientia Iranica
SCIENTIA
I RAN ICA

\title{
Development of a genetically tuned fuzzy dynamic model for nonlinear dynamical systems: Application to reaction section of Tennessee Eastman process
}

\author{
M.H. Eghbal Ahmadi ${ }^{a}$, S.J. Royaee ${ }^{a, *}$, Sh. Tayyebi ${ }^{a}$, and \\ R. Bozorgmehry Boozarjomehry ${ }^{b}$ \\ a. Petroleum Refining Technology Development Division, Research Institute of Petroleum Industry, Tehran, Iran. \\ b. Department of Chemical and Petroleum Engineering, Sharif University of Technology, Tehran, Iran.
}

Received 8 October 2017; received in revised form 19 March 2018; accepted 18 June 2018

KEYWORDS
Mamdani fuzzy
modeling;
Genetic algorithm;
Dynamic modeling;
Tennessee Eastman
process;
Optimization.

\section{Introduction}

There are two major approaches to the use of Fuzzy Inference System (FIS), namely Mamdani [1] and TakagiSugeno (TS) [2]. They have found many applications for steady state or dynamic modeling and control purposes in chemical as well as biological processes [3-9] as alternative approaches to the conventional mechanistic modeling approach.

\footnotetext{
*. Corresponding author. Tel.: +982148255103; Fax: +982144739712

E-mail address: royaeesj@ripi.ir (S.J. Royaee)
}

One of the main limitations in dealing with developing a TS-type model is the requirement of adequate data to come up with the crisp expression used as the consequences of the production rules and their corresponding parameters [10]. In addition, each TS-type model is a local approximator and the predictability of the model is valid for the specific operating conditions under which the model is developed and tested [11]. Accordingly, it can hardly be applied for analyzing the process and therefore, it is less useful for industrial practice. In contrast, there is no need for data when developing a Mamdani-type model; yet, if the data is available, the Mamdani-type fuzzy model can be optimized to increase the accuracy of prediction in a semi-quantitative manner. In addition, one can recog- 
nize that the Mamdani-type fuzzy model demonstrates several other interesting features compared to TS-type. It is more intuitive, transparent, and interpretable [12], and the knowledge of the expert dealing with the process can be easily incorporated into the model.

Despite the capabilities, the Mamdani-type fuzzy model suffers from some shortcomings. One of its main shortcomings is the lack of accuracy when it deals with high-dimensional or poorly understood systems. This shortcoming is one of the reasons why most of the researchers use the TS-type fuzzy model instead, losing the capabilities of the Mamdani approach. The estimation of the minimum miscible pressure in gas injection process [13], modeling virus removal from water using microfiltration membrane [14], prediction of diffusion coefficients in the caffeine release from hydrogel colloidosomes [15], and fuzzy estimation of the temperature distribution of furnace inner surface [16] are among limited reported applications of Mamdani approach for modeling purposes in chemical engineering.

The accuracy improvement of Mamdani-type models preserving good interpretability, which has found wide applicability in the fuzzy logic research community, can be handled using the existing optimization algorithms [17]. Merging of Mamdanitype fuzzy model and genetic (or, in general, evolutionary) algorithms can provide an opportunity to alleviate their shortcomings and capitalize on their strengths [18]. The role of GA is recognized in the optimal parameters estimation of the fuzzy model, such as the parameters of scaling functions and the universes of discourse [19,20] or the Membership Functions (MFs) [21,22]. GA is also applied as a method for rule reduction/selection by removing some rules like redundant, unnecessary, or misleading ones [21] when dealing with high-dimensional problems in which the number of rules is so large that cannot be managed efficiently. Such a problem has also been formulated as a multi-objective optimization problem in the literature to reduce the number of rules while the accuracy of the obtained model is maximized [22].

A high-performance Mamdani fuzzy model should have optimal linguistic discretization of the variables in their universe of discourse as well as correct rules defined according to true knowledge about all aspects of the underlying system. Neither one can guarantee the performance of the resulting model alone. They both must be considered in the optimization formulation of the problem. This issue is addressed in the present study by proposing a novel genetically tuned fuzzy method through which the shape of MFs (concerning both input and output variable MFs in the coding) along with the production rules composing the inference system of a startup version of the model are optimized such that it can predict the dynamic behav- ior of the process accurately enough, using a binarycoded GA. The heuristic derived from the chemical engineering knowledge is the only required information utilized in the development of the startup version of the model of the underlying process, thanks to Mamdani approach capabilities.

The GA problem is formulated such that it includes both integer and continuous decision variables leading to mixed integer nonlinear optimization problem [23]. In addition, the optimization problem is subjected to many inequality and integer constraints preserving the interpretability of the model. The proposed method is used to predict the dynamic behavior of the reaction section of the Tennessee Eastman (TE) process, which is a challenging benchmark.

The rest of the paper is organized as follows. In Section 2, Mamdani fuzzy inference method is briefly described. Afterwards, Section 3 presents the proposed genetically tuned fuzzy modeling approach, which is then followed by Section 4, in which the selected case study is introduced. The implementation of the proposed method for the selected case study and its obtained results along with their comparisons with their corresponding data of TE benchmark are explained in Section 5. Section 6 concludes the paper.

\section{Mamdani fuzzy inference}

The process of construction of a mapping from the given inputs to the desired outputs using fuzzy logic is called fuzzy inference that can be used for development of the fuzzy model. Mamdani fuzzy inference expects the output MFs to be fuzzy sets and involves main pieces of selecting MFs, constructing production rules, applying logical operations, and defuzzification [24].

The performance of a Mamdani fuzzy model depends upon many factors, among which the following are very important:

a) The type and parameters of MFs of the fuzzy sets assigned to the variables existing in the model;

b) The degree of the expertise according to which the rule table is defined.

The rule table corresponds to the governing phenomena leading to the behavior of the system. These rules that commonly consider linguistic variables in the consequence (to finally provide real-valued outputs) are written as follows for an $n$-input and $m$-output system:

$$
\begin{aligned}
& \text { If } X_{1} \text { is } A_{1} \text { and } \cdots \text { and } X_{n} \text { is } A_{n}, \\
& \text { then } Y_{1} \text { is } B_{1} \text { and } \cdots \text { and } Y_{m} \text { is } B_{m}
\end{aligned}
$$

(with $C F=w)$,

where $X_{i}$ and $Y_{i}$ are the fuzzy model linguistic input and output variables, respectively, and $A_{i}$ and $B_{i}$ are 
the linguistic values associated with the fuzzy sets specifying their meaning. The number of linguistic values of $B_{i}$ is equal to the number of fuzzy sets assigned to $Y_{i}$ that can be represented by integer numbers ranging from 1 to the maximum number of fuzzy sets assigned to $Y_{i}$, while from this point on, they are called consequence indices. Moreover, $C F$ (Certainty Factor) is a number between zero and one that weights the certainty of the rule by the value of $w$. The rule is definitely true when $w$ is equal to one, but if it is not a well-established rule widely accepted by the experts of such a system, it is less than one.

In order to enhance the performance of a Mamdani fuzzy model, both the fuzzy sets characterizing the linguistic discretization of the variables and the production rules must be optimized.

However, choosing MFs with optimum parameters is not an easy task. Sometimes, this is based on the time-consuming trial and error process. In addition, defining optimum rules that accurately present the system under investigation completely depends upon the expertise of the system to be modeled. In complex cases or in the cases that some aspects of the phenomena under study are poorly understood, expressing the phenomena by rules is difficult and, as a result, consequence indices of some rules may be incorrect. These wrong or badly defined rules (hereinafter called uncertain rules) worsen the model prediction capability when co-existing with other rules.

\section{Genetically tuned fuzzy modeling method}

The proposed genetically tuned Mamdani fuzzy modeling method consists of two main steps: (i) Constructing a startup version of the model using only the heuristic knowledge; and (ii) Tuning the procedure using the genetic algorithm. In the first step, the variables determining the dynamic behavior of the system are defined. Given that, the input variables which affect the selected output variables are determined. The recurrent fuzzy model [25] is used, which is a mapping of past inputs and outputs to the future outputs. By doing so, the previously calculated sampling times of the output variables are also considered as the input variables of the model. As a result, the transient behavior of the model can be well predicted. Afterwards, a base fuzzy model is defined in which the fuzzy sets of the variables are chosen to be evenly distributed throughout their corresponding universes of discourse. The production rules constituting the rule table of the starting model are also set up based on the knowledge and expertise of the experts who have been working with the system. These rules are constructed to present all feasible combinations of the input variables of the model. Among them, there are vague or complex aspects, especially in complex nonlinear systems, where there is no sufficient knowledge, leading to uncertain rules. Even there may be some wrong rules among uncertain rules, leading to opposite prediction of the model in some time intervals. This model is used as the startup version of the model which has to be tuned. The only required information to construct the startup version of the model is the heuristic derived from chemical engineering knowledge and the analysis of the underlying system, bypassing solving a couple of the differential and algebraic mathematical equations governing the dynamic behavior of the process.

In the second step, using a new method, a binarycoded GA is formulated for simultaneous optimization of both parameters that characterize the linguistic definition of the variables in the model (MFs of the fuzzy model variables) and consequence indices (or output linguistic values) of the uncertain rules of the system.

For the sake of simplicity, all the variables existing in the system are represented by three fuzzy sets whose MFs are chosen to be the most frequently used ones, which are the triangular ones for the middle fuzzy set and half trapezoidal ones for the sets existing at the extremes of the domains of variables. Selection of these types of MFs is due to the fact that they are very simple, and therefore, the calculation of the output values is computationally less demanding. This decreases the required time to obtain the optimum values of the decision variables of the optimization problem [26]. The most important drawback of triangular and trapezoidal MFs is that they are non-differentiable in their corner points and consequently, they may lead to problems if using classical gradient-based optimization algorithms. However, when evolutionary algorithms are used as the optimization method, this drawback does not come into the picture at all.

The triangle MF is specified by three parameters $\{a, b, c\}$ as follows:

$$
f(x)= \begin{cases}0, & x \leq a \\ \frac{x-a}{b-a}, & a \leq x \leq b \\ \frac{c-x}{c-b}, & b \leq x \leq c \\ 0, & x \geq c\end{cases}
$$

Likewise, trapezoidal MF is specified by four parameters $\{a, b, c, d\}$ as follows:

$$
g(x)= \begin{cases}0, & x \leq a \\ \frac{x-a}{b-a}, & a \leq x \leq b \\ 1, & b \leq x \leq c \\ \frac{d-x}{d-c}, & c \leq x \leq d \\ 0, & x \geq d\end{cases}
$$

\subsection{Tuning of fuzzy model as an optimization problem}

The formulation of the problem is a typical Mixed Integer Non-Linear Programming (MINLP) optimization 
as follows:

$\min _{v . w}$ Obj.Fun : $M A P E$

$$
=\frac{100}{a \times b} \sum_{j=1}^{b} \sum_{i=1}^{a}\left|\frac{y_{i, j}(m)-y_{i, j}(p)}{y_{i, j}(m)}\right|,
$$

s.t. $v \in V \in[01]$,

$$
w \in W \in\{-1,0,1\}
$$

where $a$ is the number of data points, $b$ is the number of model output variables, and $M A P E$ is the mean absolute percentage error that is defined according to the difference between $y(m)$ (the vector of measured data) and $y(p)$ (the vector of model predictions data). In addition, $v$ and $w$ are continuous (MF parameters) and integer (consequence indices) decision variables, respectively. All input and output data of the variables of the model are normalized with all variables being between 0 and 1 . Therefore, all the parameters that characterize the MFs of the model variables are real values between 0 and 1 .

MINLP inherently contains many solution candidates and is a non-convex and complex problem [27]. Being different from gradient-based methods, GA requires only the information about the values of the objective function that can effectively tackle MINLP [28] with reduced chances of being trapped in local optima. Furthermore, the restrictions imposed on the conventional optimization method, such as the need for linearization of non-linear terms of the formulation, are all relaxed. The solution to MINLP problems with GA is a valid approach in non-convex problems in which computational time is not of primary concern [29].

\subsection{Chromosomal representation of the optimization problem}

Each chromosome of GA is encoded such that it is composed of two parts of a certain length, which is schematically shown in Figure 1.

The first part is encoded to determine the parameters of the triangular and trapezoidal MFs $\left(a_{i}\right.$, $b_{i}, c_{i}$, and $d_{i}$ ). The second part corresponds to the consequence indices of the uncertain rules.

As mentioned, not all the combinations of input variables are completely understood by the expert. The lack of knowledge in each combination of the input variables can be characterized by the $C F$ values corresponding to the rules.
Among all the rules of the inference system, the consequence indices of those whose $C F$ has been set to a value lower than a threshold (say 0.8) are chosen to be optimized through optimization procedure. The threshold value of 0.8 is completely a case dependent value that is defined according to the desired level of accuracy from the optimum model. Needless to say, the larger the threshold value is defined, the larger the portion of the rule consequence indices is considered to be optimized through the optimization problem that leads to the higher accuracy of the obtained model at the expense of increasing the chromosome length.

\subsection{Constraints of the optimization problem}

The search performed by the GA is subject to inequality and integer constraints. Three types of inequality constraints are put on the parameters of the MFs concerning their semantic and interpretability properties as shown in Figure 2.

The first type is the constraints that try to keep the shape of triangular and trapezoidal MFs. For instance, the " $a$ " parameter should not be larger than the " $b$ " parameter in a triangular MF shape. The second type is the constraints that try to keep the fuzzy sets semantic such that the center point (that is, " $b$ " for triangular, " $c$ " for the trapezoidal assigned to the first fuzzy set, and " $b$ " for the trapezoidal assigned to the third fuzzy set) of each fuzzy set would not be greater than the center point of the subsequent linguistic terms. The third type is the constraints that force at least two subsequent fuzzy sets to cross each other.

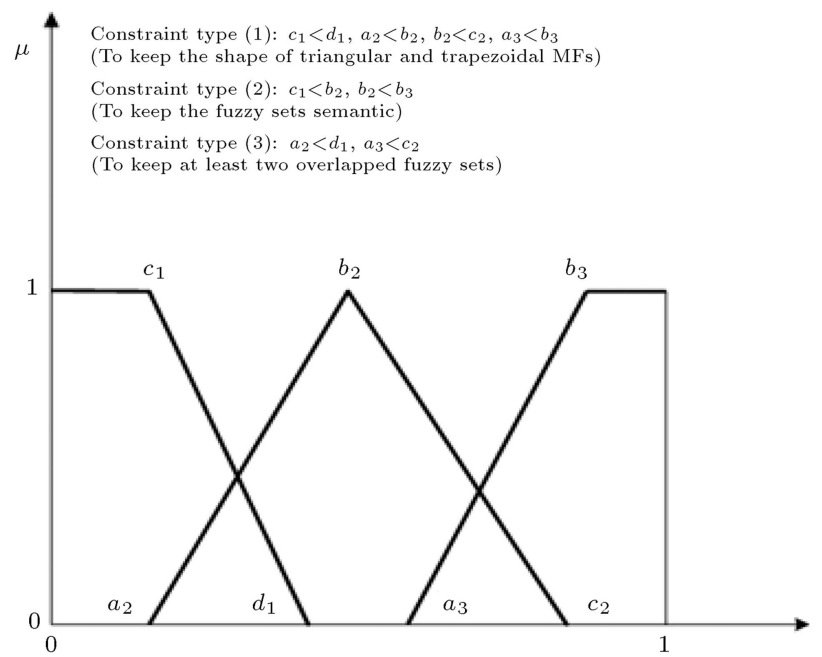

Figure 2. Constraints on MF parameters of the fuzzy sets in the genetic tuning process.

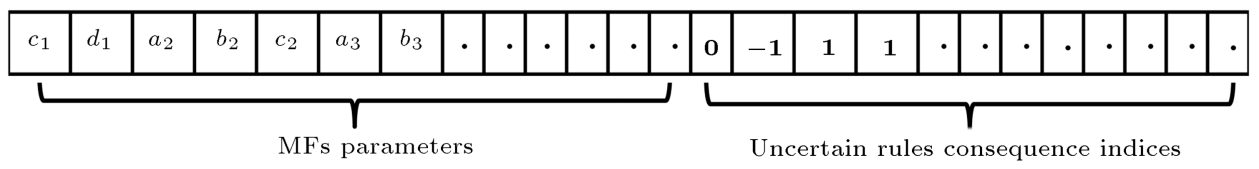

Figure 1. A chromosome sample of GA in this work. 
In addition to the above inequality constraints, the consequence indices that are decoded and taken from the second part of the chromosome are constrained to be an integer number, whether $-1,0$, or 1. Zero implies that the initial index defined for the corresponding uncertain rule in the startup version of the model does not need to change while "1" or " -1 " means that it should be added by " 1 " or " -1 ", respectively, to make modification in its prediction capability. It is worth mentioning that making larger modifications in the inference system with very uncertain rules through decoding the corresponding genome of the second part of the chromosome may result in 5 integer numbers to be obtained, i.e., whether $-2,-1,0$, 1 , or 2. In other words, the lower and upper bounds of the integer values representing the consequence indices completely depend upon the degree of uncertainty of the defined rules, which are characterized by the threshold value of $C F$ assigned to the rules. The more uncertain the rules, the lower the threshold value is recommended to be assigned. The resulting optimum consequence indices are constrained to be at least one and at most equal to the number of fuzzy sets of the corresponding output variable, which is 3 in the model.

As described, the consequence indices taken from the chromosome have a limited number of candidate values (for instance, 3 or 5 ) that significantly reduce the search space, and thus guarantee obtaining the optimum consequence indices of the uncertain rules. Accordingly, all vague aspects of the model can be treated.

\section{Case study: The reaction section of TE process}

Tennessee Eastman process is a plant-wide process control benchmark introduced by Downs and Vogel [30] to serve as a test-bed for the purpose of studying new technologies for process control, modeling, monitoring, fault diagnosis, and other potential applications. It consists of a mixed reactor (reaction section), a product condenser, a vapor-liquid separator, and a product stripper. The present work is focused on its reaction section, which is shown in Figure 3.

In the reaction section, the reactants $A, C, D$, and $E$ are fed to the reactor, in which the following exothermic reactions take place:

$$
\begin{aligned}
& A(g)+C(g)+D(g) \rightarrow G(\text { liq }), \quad \text { Product } 1, \\
& A(g)+C(g)+E(g) \rightarrow H(\text { liq }), \quad \text { Product } 2, \\
& 1 / 3 A(g)+D(g)+1 / 3 E(g) \rightarrow F(\text { liq }), \quad \text { Byproduct. }
\end{aligned}
$$

The reactor is agitated and has an internal cooler to remove the heat of the reactions. The products are discharged from the reactor along with the non-reacted

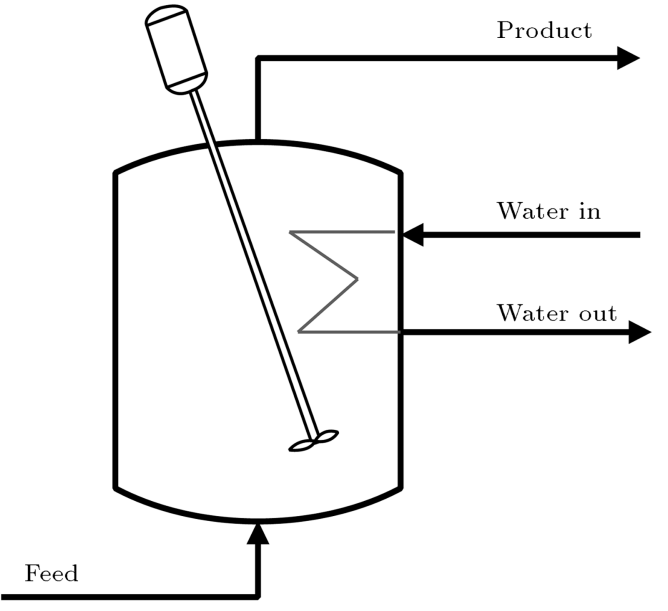

Figure 3. Reaction section in TE process.

reactants and then, they pass through a condenser to make a two-phase stream consisting of gas and liquid phases, which goes to the subsequent separation section. The nonlinear nature of the plant is mainly due to the reactions occurring in the reactor.

The process has the total of 41 measured variables, of which 22 variables are continuous and 19 variables are sampled process measurements. These measurements include pressure, temperature, flowrate, molar composition of some streams, and liquid levels of the equipment. Besides, 12 manipulated variables are included in this process to be used for different dynamic excitations or control purposes. The detailed description of the TE process can be found in [30].

Using the described genetically tuned fuzzy method is aimed at developing an optimum Mamdani fuzzy model to predict the dynamic behavior of the reaction section of the TE process. The main operating variables characterizing the dynamic behavior of the reaction section are the reactor temperature, pressure and level, and the outlet cooling water temperature in the internal cooler. According to the chemical engineering knowledge, the first three variables are mostly affected and determined by the set of reactions taking place in the reactor and heat exchange against the cooling water of the internal cooler. Furthermore, the outlet temperature of the cooling water in the internal cooler is determined through heat exchange against the reactor content. As a result, with regard to the manipulated or measured variables defined in the TE process (Table 1), feed conditions including feed flow and molar composition; agitator speed, which affects the overall heat transfer [30]; and cooling water flow are taken into consideration as the input variables of the model.

The schematic of the fuzzy model is depicted in Figure 4.

Having defined the input/output variables of the fuzzy model as well as their linguistic definitions, the 
Table 1. Measured and manipulated variables of the reaction section in TE.

\begin{tabular}{ll}
\hline Reactor feed rate $-F_{\text {feed }}$ (measured) & $A$ molar composition in feed - $Z_{A}$ measured) \\
Reactor pressure $-P_{\text {reactor }}$ (measured) & $B$ molar composition in feed - $Z_{B}$ (measured) \\
Reactor level - $L_{\text {reactor }}$ (measured) & $C$ molar composition in feed - $Z_{C}$ (measured) \\
Reactor temperature $-T_{\text {reactor }}$ (measured) & $D$ molar composition in feed - $Z_{D}$ (measured) \\
Reactor cooling water outlet temperature - Tout $t_{\text {cw-reactor }}$ (measured) & $E$ molar composition in feed - $Z_{E}$ (measured) \\
Reactor cooling water flow - $F_{\text {cw-reactor }}$ (manipulated) & $F$ molar composition in feed - $Z_{F}$ (measured) \\
Reactor agitator speed - Ag.Speed (manipulated) &
\end{tabular}

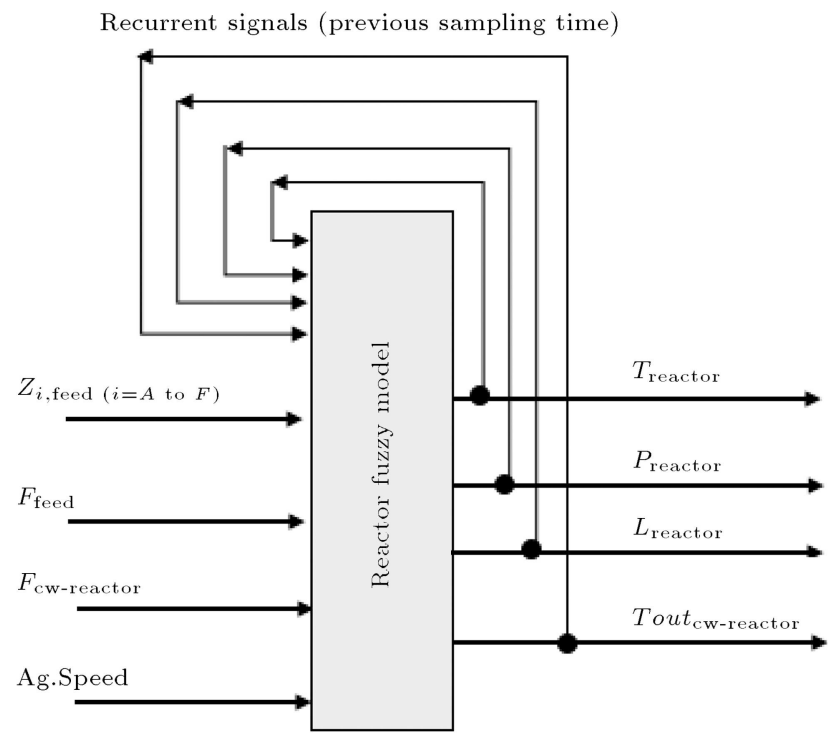

Figure 4. The fuzzy model scheme of the reaction section in TE process.

rules that govern the fuzzy model are defined in the next step to construct the startup version of the model.

As an example of these rules, suppose that at the current sampling time, the feed has the normal amount of raw material, the cooling water flow is high, and the value of the reactor temperature is low, while the other inputs are medium; thus, the reactor is expected to have low temperature in the next sampling time. As another example, suppose that at the current sampling time, the feed has a high amount of raw material, the cooling water flow is low, and value of the reactor temperature is high, while the other inputs are medium; thus, the reactor is definitely expected to have high temperature in the next sampling time and the cooling water outlet temperature will be high if its previous sampling time temperature is medium or high.

Likewise, the table of rules can be created using such a heuristic analysis derived from chemical engineering knowledge about the model, without considering the relation of reaction rate, the relation of heat transfer coefficient, etc. This capability is unique to Mamdani-type fuzzy approach, in which the qualitative translation of the governing phenomenon of the underlying system is incorporated in the model, bypassing the complexity of solving various types of mathematical equations used in conventional dynamic modeling methods.

The uncertain rules and the shape of fuzzy sets will be optimized by application of genetic algorithm. The inference table consists of 1458 rules, of which 59 have $C F$ s lower than 0.8 (uncertain rules), that can be considered as integer decision variables. In addition, the model has 12 input/output variables, each having 7 parameters to be optimized; thus, the number of MF decision variables is 84 .

\section{Results and discussion}

The code provided by Downs and Vogel [30] is used to test and validate the developed optimized model for a variety of operating conditions. Using a random method, which is called "Random Number Random Interval-RNRI," the dataset is generated by stimulating the manipulated variables of the TE, and the dynamic data corresponding to the reaction section are collected. RNRI is a specific algorithm to generate step-wise random excitation pattern in manipulated variables of the TE process, simultaneously. Having set the plant initial condition as steady-state values provided by Downs and Vogel [30], two random numbers are generated for each manipulated variable during the dynamic run of the plant. The first random number (say, MVrandom1) is a value inside the 0 and 100 limits for one of the manipulated variables. The second random number is a time (say, trandom 1 ) during which the corresponding manipulated variable is set to the MVrandom1. After a time interval of trandom1, another random number is generated by the RNRI (say, MVrandom2) that is set to the corresponding manipulated variable during another randomly generated time interval (say, trandom2). Likewise, all other 11 manipulated variables are excited simultaneously and independently using this algorithm during the transient operation of the plant. The generated step-wise RNRI values of the two selected manipulated variables (reactor cooling water flow-rate and, simultaneously, the reactor agitator speed) of the reaction section are shown in Figure 5.

With this process stimulating procedure, the gen- 
Table 2. MAPE of the output variables of the startup version and optimized fuzzy models*.

\begin{tabular}{lcccc}
\hline & $\boldsymbol{T}_{\text {reactor }}$ & $\boldsymbol{P}_{\text {reactor }}$ & $\boldsymbol{L}_{\text {reactor }}$ & $\boldsymbol{T}_{\boldsymbol{T}} \boldsymbol{o u t}_{\text {cw-reactor }}$ \\
\hline MAPE (startup version of the model) & $6.56 \%$ & $2.15 \%$ & $8.36 \%$ & $8.79 \%$ \\
MAPE (optimized model) & $1.17 \%$ & $0.38 \%$ & $1.50 \%$ & $1.57 \%$ \\
\hline
\end{tabular}

${ }^{*}$ The overall accuracy of optimized model is $98.84 \%$.

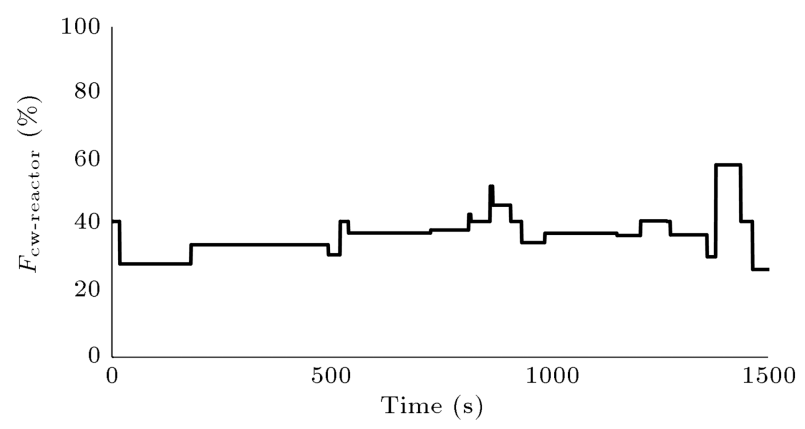

(a)

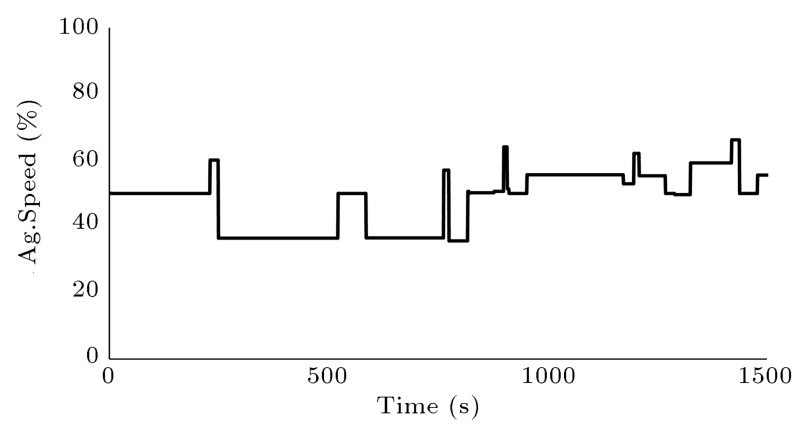

(a)

Figure 5. The RNRI generated value for (a) reactor cooling water flow rate, and (b) the reactor agitator speed.

erated data set embraces almost all characteristics of the whole process so that it would be rich enough to capture all dynamic modes of the reaction section of the TE process. It should be noted that the generated data are not beyond the data process shutdown limits defined in the TE plant-wide code.

A portion of data is used to develop the optimized fuzzy model and the rest is kept aside for validation purposes. Two stopping criteria have been used in GA, which are as follows:

1. The maximum number of generations (100) is reached;

2. The best solution during the evolution process does not change to a better value.

The algorithm stops when one of the stopping criteria is met. The optimal Mamdani fuzzy model is obtained after 30 generations.

The optimum shapes of fuzzy set MFs for the input and output variables of the developed fuzzy model are presented in Figure 6. In addition, the consequence indices of the rules in the model that have
$C F$ s lower than 0.8 (uncertain rules) are optimized through the genetically tuned fuzzy method.

The open-loop dynamic responses for four outputs of the fuzzy model and their corresponding process measurements in the TE code for startup version of the fuzzy model (before optimization) as well as the optimized model are shown in Figure 7.

The randomly excited manipulated variables cause different dynamic changes in the output variables of the model. As graphically observed in Figure 7, the startup version of the model constructed only by the heuristic analysis of the reaction section demonstrates almost the same general trends with $\mathrm{TE}$ data. It should be noted that even if there were some wrong rules among uncertain rules, opposite prediction of the model might emerge in some time intervals. The predictability of the startup version of the model is enhanced in the optimized fuzzy model characterized by correct optimum rules and optimized shapes of the fuzzy sets presenting the linguistic definitions of the variables. As a result, the dynamic behavior of the reaction section is satisfactorily captured, showing that the proposed method performs quite appropriately so that the outputs of the fuzzy model almost coincide with their corresponding values that are obtained by the TE simulator proposed by Downs \& Vogel [30]. For instance, with increase in the reactor temperature due to several reasons such as low agitator speed and cooling water flow or high amount of raw materials in the feed, in general, the cooling water outlet temperature also increases. In addition, the high reactor temperature accelerates the reactions in favor of more conversion of gas reactants to liquid products and vaporization of the liquid level. This can reduce the reactor pressure as well as its level. These dynamic changes are fairly captured by the optimized fuzzy model.

Table 2 shows a comparison between the outputs of the fuzzy model (for both the startup version and the optimized models) and their corresponding values provided by the TE code.

\section{Conclusion}

A novel method to optimally develop a fuzzy model based on Mamdani approach for predicting the dynamic behavior of a process was proposed. The proposed method consisted of two main steps: 

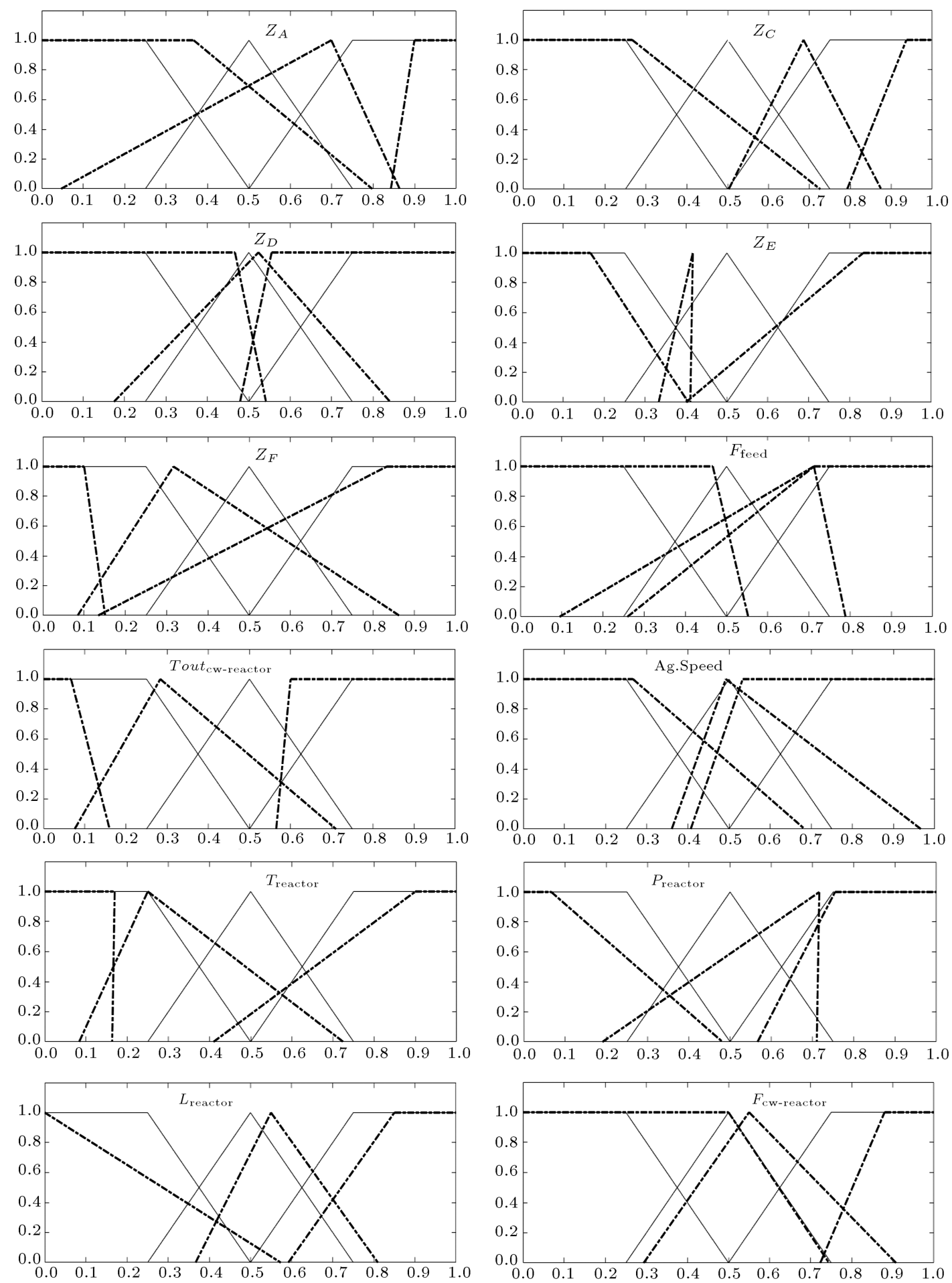

Figure 6. Initial (solid lines) and optimized (dash lines) fuzzy sets of the input and output variables of the model.

i) Constructing a startup version of the model;

ii) Tuning procedure using the genetic algorithm.

In the first step, the only required information was the heuristic derived based on the chemical engineering knowledge about the process, bypassing solving various types of mathematical equations governing the process dynamic behavior. The input/output variables as well as production rules of the startup version of the fuzzy model were selected according to the heuristic knowledge about the governing phenomena with regards to what was defined by Downs \& Vogel [30]. In the second step, the optimal estimation of the parameters of the startup version of the fuzzy model was formulated as a 

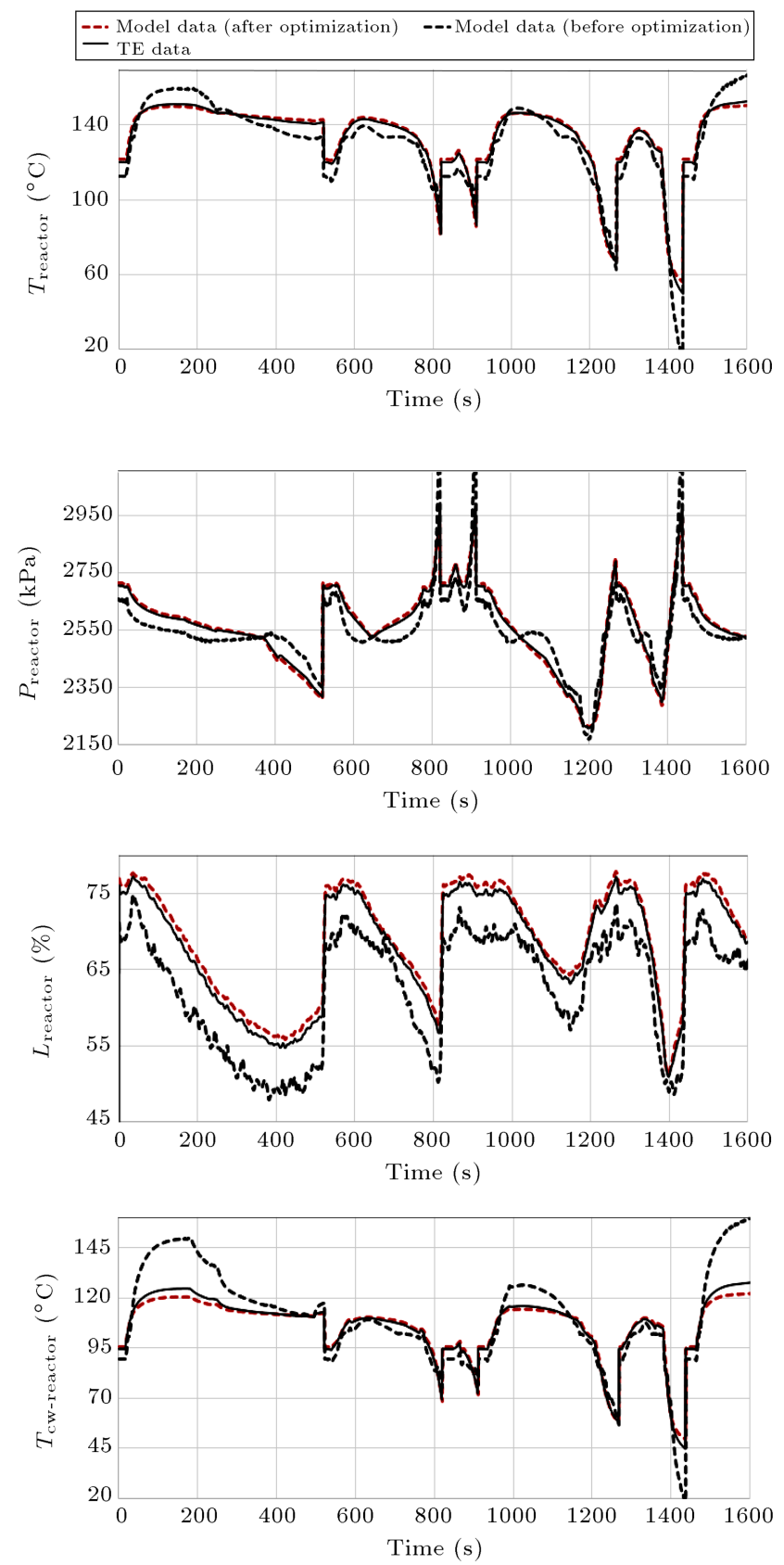

Figure 7. Observed TE and optimized fuzzy model data for the reaction section for randomly excited input data.

constrained MINLP problem and carried out. Integer decision variables of the MINLP problem were the consequence indices of uncertain rules in the fuzzy inference system rule-table. These rules corresponded to the operating conditions of the process or those combinations of input variables for which there was no sufficient knowledge or poor understanding was in hand. Continuous decision variables of the MINLP problem were the parameters that characterized the MFs corresponding to fuzzy sets of the input and output variables of the fuzzy model. The performance of the proposed method was evaluated by its application in modeling the reaction section of the TE process. The optimal fuzzy model was obtained and used to predict the transient behavior of temperature, pressure, and level of the reactor as well as the reactor cooling water outlet temperature, with calculated MAPEs of $1.17 \%, 0.38 \%, 1.5 \%$, and $1.57 \%$, respectively. The obtained results showed the appropriate performance and high accuracy of the proposed genetically tuned fuzzy method for dynamically modeling of TE reaction section.

\section{References}

1. Mamdani, E.H. and Assilian, S. "An experiment in linguistic synthesis with a fuzzy logic controller", Int. J. Man. Mach. Stud., 7(1), pp. 1-13 (1975).

2. Takagi, T. and Sugeno, M. "Fuzzy identification of systems and its applications to modeling and control", IEEE Trans. Syst. Man Cybern., 15(1), pp. 116-132 (1985).

3. Kandi-D, M., Soleymani, M., and Ghadimi, A.A. "Designing an optimal fuzzy controller for a fuel cell vehicle considering driving patterns", Sci. Iran., 23(1), pp. 218-227 (2016).

4. Lima, N.M.N., Liñan, L.Z., Manenti, F., Maciel Filho, R., Maciel, M.R.W., Embiruçu, M., and Medina, L.C. "Fuzzy cognitive approach of a molecular distillation process", Chem. Eng. Res. Des., 89(4), pp. 471-479 (2011).

5. Lam, H.K. "A review on stability analysis of continuous-time fuzzy-model-based control systems: From membership-function-independent to membership-function-dependent analysis", Eng. Appl. Artif Intell., 67, pp. 390-408 (2018).

6. Krzywanski, J. and Nowak, W. "Modeling of bed-towall heat transfer coefficient in a large-scale CFBC by fuzzy logic approach", Int. J. Heat Mass Transf., 94, pp. 327-334 (2016).

7. Boulkaibet, I., Belarbi, K., Bououden, S., Marwala, T., and Chadli, M. "A new TS fuzzy model predictive control for nonlinear processes", Expert. Syst. Appl., 88, pp. 132-151 (2017).

8. Kamesh, R. and Rani, K.Y. "Parameterized datadriven fuzzy model based optimal control of a semibatch reactor", ISA Trans., 64, pp. 418-430 (2016).

9. Esfandyari, M., Fanaei, M.A., and Zohreie, H. "Adaptive fuzzy tuning of PID controllers", Neural Comput. Appl., 23(1), pp. S19-S28 (2013).

10. Adoko, A.C., Gokceoglu, C., Wu, L., and Zuo, Q.J. "Knowledge-based and data-driven fuzzy modeling for rockburst prediction", Int. J. Rock Mech. Min. Sci, 61, pp. 86-95 (2013).

11. Habbi, H., Zelmat, M., and Bouamama, B.O. "A dynamic fuzzy model for a drum-boiler-turbine system", Automatica, 39(7), pp. 1213-1219 (2003). 
12. Sala, A., Guerra, T.M., and Babuška, R. "Perspectives of fuzzy systems and control", Fuzzy Sets Syst., 156(3), pp. $432-444(2005)$.

13. Ahmadi, M.A. and Ebadi, M. "Fuzzy modeling and experimental investigation of minimum miscible pressure in gas injection process", Fluid Phase Equilib., 378, pp. 1-12 (2014).

14. Madaeni, S.S. and Kurdian, A.R. "Fuzzy modeling and hybrid genetic algorithm optimization of virus removal from water using microfiltration membrane", Chem. Eng. Res. Des., 89(4), pp. 456-470 (2011).

15. Amiryousefi, M.R., Mohebbi, M., Golmohammadzadeh, S., Koocheki, A. and Baghbani, F. "Fuzzy logic application to model caffeine release from hydrogel colloidosomes", J. Food Eng., 212, pp. 181-189 (2017).

16. Wang, G., Luo, Z., Zhu, L., Chen, H., and Zhang, L. "Fuzzy estimation for temperature distribution of furnace inner surface", Int. J. Therm. Sci, 51(1), pp. 84-90 (2012).

17. Cordón, O., Gomide, F., Herrera, F., Hoffmann, F., and Magdalena, L. "Ten years of genetic fuzzy systems: Current framework and new trends", Fuzzy Sets Syst., 141(1), pp. 5-31 (2004).

18. Herrera, F. "Genetic fuzzy systems: Taxonomy, current research trends and prospects", Evol. Intell., 1(1), pp. 27-46 (2008).

19. Gudwin, R., Gomide, F., and Pedrycz, W. "Context adaptation in fuzzy processing and genetic algorithms", Int. J. Intell. Syst., 13(10-11), pp. 929-948 (1998).

20. Cordón, O., Herrera, F., Del Jesus, M.J., Magdalena, L., Sánchez, A.M., and Villar, P. "A multiobjective genetic algorithm for feature selection and granularity learning in fuzzy-rule based classification systems", 20th NAFIPS Int. Conf., 3, Vancouver, Canada, pp. 1253-1258 (2001).

21. Cordón, O., José del Jesus, M., and Herrera, F. "Genetic learning of fuzzy rule-based classification systems cooperating with fuzzy reasoning methods", Int. J. Intell. Syst., 13(10-11), pp. 1025-1053 (1998).

22. Pulkkinen, P. and Koivisto, H. "A dynamically constrained multiobjective genetic fuzzy system for regression problems", IEEE Trans. Fuzzy Syst., 18(1), pp. 161-177 (2010).

23. Harmsen, G.J. "Industrial best practices of conceptual process design", Chem. Eng. Process. Process Intensif., 43(5), pp. 677-681 (2004).

24. Zimmermann, H.J. "Fuzzy set theory", Wiley Interdisciplinary Reviews: Comput. Stat, 2(3). pp. 317-332 (2010).
25. Gorrini, V. and Bersini, H. "Recurrent fuzzy systems", 3rd IEEE World Conf. on Comput Intell., New Rochelle, NY, USA, pp. 193-198 (1994).

26. Pedrycz, W. "Why triangular membership functions?", Fuzzy Sets Syst., 64(1), pp. 21-30 (1994).

27. Sharifzadeh, M. "Integration of process design and control: A review", Chem. Eng. Res. Des., 91(12). pp. 2515-2549 (2013).

28. Wasanapradit, T., Mukdasanit, N., Chaiyaratana, N., and Srinophakun, T. "Solving mixed-integer nonlinear programming problems using improved genetic algorithms", Korean J. Chem. Eng., 28(1), pp. 32-40 (2010).

29. Costa, L. and Oliveira, P. "Evolutionary algorithms approach to the solution of mixed integer non-linear programming problems", Comput. Chem. Eng., 25(23), pp. 257-266 (2001).

30. Downs, J.J. and Vogel, E.F. "A plant-wide industrial process control problem", Comput. Chem. Eng., 17(3), pp. 245-255 (1993).

\section{Biographies}

Mohammad Hosein Eghbal Ahmadi received the $\mathrm{BS}, \mathrm{MS}$, and $\mathrm{PhD}$ degrees in Chemical Engineering from Tehran University, Sharif University of Technology, and Research Institute of Petroleum Industry, respectively. He has been working on modeling, simulation, and optimization of chemical processes during the recent years.

Sayed Javid Royaee is an Assistant Professor at Research Institute of Petroleum Industry in Iran. He received MS and $\mathrm{PhD}$ degrees in Chemical Engineering from Amirkabir University of Technology in Iran. His research interests include process modeling and simulation, kinetic modeling, and artificial intelligence.

Shokoufe Tayyebi is an Assistant Professor at Research Institute of Petroleum Industry in Iran. She received BS, MS, and $\mathrm{PhD}$ degrees in Chemical Engineering from Sharif University of Technology in Iran. Her research interests include modeling and simulation, process control, and artificial intelligence.

Ramin Bozorgmehry Boozarjomehry is a Professor of Chemical Engineering at Sharif University of Technology. He received $\mathrm{BS}, \mathrm{MS}$, and $\mathrm{PhD}$ degrees in Chemical Engineering from Tehran University, Sharif University of Technology, and University of Calgary, respectively. His research interests are modeling and simulation, process control, and artificial intelligence. 